\title{
A I0-year review of underlying diseases for endothelial keratoplasty (DSAEK/DMEK) in a tertiary referral hospital in Japan
}

This article was published in the following Dove Press journal:

Clinical Ophthalmology

\section{Tsubasa Nishino \\ Akira Kobayashi \\ Hideaki Yokogawa \\ Natsuko Mori \\ Toshinori Masaki \\ Kazuhisa Sugiyama}

Department of Ophthalmology, Kanazawa University Graduate School of Medical Science, Kanazawa, Japan
Correspondence: Akira Kobayashi Department of Ophthalmology, Kanazawa University Graduate School of Medical Science, I3-I Takara-Machi, Kanazawa-Shi, Ishikawa-ken 920-864I, Japan

Tel +8I 762652403

Fax +8I 762229660

Email eyekoba@gmail.com
Purpose: To report a 10-year review of endothelial keratoplasty (EK) procedures, Descemet's stripping automated endothelial keratoplasty (DSAEK) and Descemet's membrane endothelial keratoplasty (DMEK), and underlying diseases at a tertiary referral hospital in Japan.

Study design: A single-center, retrospective case series.

Methods: We retrospectively reviewed all medical records of bullous keratopathy (BK) surgically treated by EK (DSAEK/DMEK) at Kanazawa University Hospital from January 2007 to December 2016. Changes or modifications to the annual number of EK procedures and underlying diseases were analyzed.

Results: During this period, 320 EK procedures (DSAEK: 288 cases, DMEK: 32 cases) were performed on 250 patients. Total annual EKs gradually increased from 19 to 45 cases between 2007 and 2016. The annual number of DSAEKs was stable, although the proportion of DSAEKs to other procedures decreased significantly as re-DSAEKs and DMEKs increased. BK after argon laser iridotomy (ALI) was the leading cause in 2007, followed by Fuchs' endothelial dystrophy (FED) and failed penetrating keratoplasty. In 2016, BK after trabeculectomy (TLE) was most prevalent, followed by failed DSAEK, failed penetrating keratoplasty, and pseudophakic BK. The decreased ALI and FED, and increased BK after TLE and failed DSAEK were statistically significant.

Conclusion: The distribution of EK procedures (DSAEK/DMEK) and underlying diseases changed over 10 years at a tertiary referral hospital in Japan. The proportion of re-DSAEK and DMEK increased among all EK procedures. Most significantly, among the underlying diseases, decreased ALI and FED and increased TLE and failed DSAEK were observed. Extended multicenter analysis may further elucidate the changes in EK procedures and the causes of BK in Japan.

Keywords: argon laser iridotomy, trabeculectomy, reoperation, Fuchs' endothelial dystrophy

\section{Introduction}

Descemet's stripping automated endothelial keratoplasty (DSAEK) ${ }^{1-7}$ and Descemet's membrane endothelial keratoplasty (DMEK) have been performed worldwide as the preferential treatment for corneal endothelial dysfunction for $>10$ years. ${ }^{8-18}$ Recent statistics have shown that these techniques are the most common keratoplasty procedures performed in the US. ${ }^{19}$ The use of precut DSAEK $^{20}$ or prestripped DMEK tissues $^{21}$ supplied by the eyebank has greatly shortened operation time, eliminating the need for a large capital investment in an expensive microkeratome machine for DSAEK, and preventing tissue loss due to unsuccessful Descemet's membrane stripping/harvesting during DMEK. 
The great majority of underlying diseases contributing to bullous keratopathy (BK) in western countries have been reported to be Fuchs' endothelial dystrophy (FED), ${ }^{15,16}$ followed by cataract surgery. ${ }^{19,22}$ In Japan, however, the reported underlying diseases are quite different compared with western countries; while Fuchs' dystrophy is rare in Japan, BK after argon laser iridotomy (ALI) is relatively common..$^{23,24}$ Therefore, it should be important and necessary to understand recent trends of endothelial keratoplasty (EK) procedures and their underlying diseases for corneal surgeons in this region or western corneal surgeons treating Japanese and/or Asian patients.

Herein, we report a 10-year review of EK procedures and their underlying diseases that were performed at a tertiary referral hospital in Japan.

\section{Methods}

The research plan was approved by the Medical Ethics Committee of Kanazawa University (approval number: 2533), and followed the tenets of the Declaration of Helsinki. We retrospectively reviewed all medical records for patients with BK surgically treated by EK (DSAEK/DMEK) at Kanazawa University Hospital from January 2007 to December 2016. Written informed consent was obtained from all participants before enrollment into this study. All EK surgeries were performed by 2 trained surgeons (AK and HY). First, the overall number of EK surgeries and procedures as well as underlying diseases during this period were recorded. Second, changes in the prevalence of the different EK procedures as well as their underlying diseases were statistically analyzed. A graft failure after penetrating keratoplasty (PK)/DSAEK/DMEK was defined as failed PK, failed DSAEK, or failed DMEK, respectively. BK due to primary failure immediately after EK was categorized as either failed DSAEK or failed DMEK. Eyes, which had undergone DSAEK (failed DSAEK) or DMEK (failed DMEK) multiple times were also enrolled independently. Pseudophakic BK (PBK) was defined as BK after phaco cataract surgery without any other apparent causes, such as exfoliation syndrome (XFS) or preexisting low endothelial cell density or history of other intraocular surgeries.

\section{Statistical methods}

To detect annual trend in causal diseases and surgical procedure of corneal endothelial transplantation in the range of 2007-2016, simple linear regression was used for 12 diseases and 4 procedures. The response variable was percentage of disease/procedure to the total number of transplantations in 1 year, and the exploratory variable was the year as continuous variable in the regression model. If $P$-value to the null hypothesis, slope for year $=0$, was $<0.05$, then the disease/procedure was considered to have a significant increasing or decreasing trend over year.

\section{Results}

Three hundred and twenty EK procedures (DSAEK: 288 cases, DMEK: 32 cases; average age: $71.5 \pm 11.1$ years old) were performed on 250 patients (105 males: 115 eyes, 144 cases; 145 females: 157 eyes, 176 cases) during this 10 -year period (Table 1). The total annual number of EKs gradually increased from 19 cases in 2007 to 45 in 2016 with an exceptional spike in 2011 (43 cases) (Figures 1 and 2).

As shown in Table 1, the leading indications for EK (DSAEK/DMEK) of the total 10-year period was ALI ( 68 cases, $21.3 \%$ ), followed by PBK (50 cases, $15.6 \%$ ), trabeculectomy (TLE) (46 cases, 14.4\%), failed DSAEK (40 cases, 12.5\%), failed PK (29 cases, 9.1\%), FED (23 cases, 7.2\%), XFS (10 cases, $3.1 \%$ ), forceps delivery ( 6 cases, $1.9 \%$ ), failed DMEK (6 cases, 1.9\%), cytomegalovirus (CMV) endotheliitis ( 5 cases, $1.6 \%$ ), iridocorneal endothelial syndrome (ICE) ( 2 cases, $0.6 \%$ ), and others ( 35 cases, $10.9 \%$ ). All of the eyes with BK after TLE were pseudophakia.

Significant annual trends were observed for 3 types of procedures, DSAEK, re-DSAEK, and DMEK. Although the total annual number of DSAEKs was stable between 2007 and 2016, the relative proportion of DSAEKs decreased significantly $(P=0.01)$. In contrast, the relative number of

Table I Underlying disease indications for DSAEK/DMEK from 2007 to 2016

\begin{tabular}{lll}
\hline Underlying disease & No of cases & Percentage \\
\hline ALI & 68 & 21.3 \\
PBK & 50 & 15.6 \\
TLE & 46 & 14.4 \\
Failed DSAEK & 40 & 12.5 \\
Failed PK & 29 & 9.1 \\
FED & 23 & 7.2 \\
XFS & 10 & 3.1 \\
Forceps delivery & 6 & 1.9 \\
Failed DMEK & 6 & 1.9 \\
CMV endotheliitis & 5 & 1.6 \\
ICE & 2 & 0.6 \\
Other & 35 & 10.9 \\
Total & 320 & \\
\hline
\end{tabular}

Abbreviations: ALI, argon laser iridotomy; CMV, cytomegalovirus; DMEK, Descemet's membrane endothelial keratoplasty; DSAEK, Descemet's stripping automated endothelial keratoplasty; FED, Fuchs' endothelial dystrophy; ICE, iridocorneal endothelial syndrome; PBK, pseudophakic bullous keratopathy; PK, penetrating keratoplasty; TLE, trabeculectomy; XFS, exfoliation syndrome. 


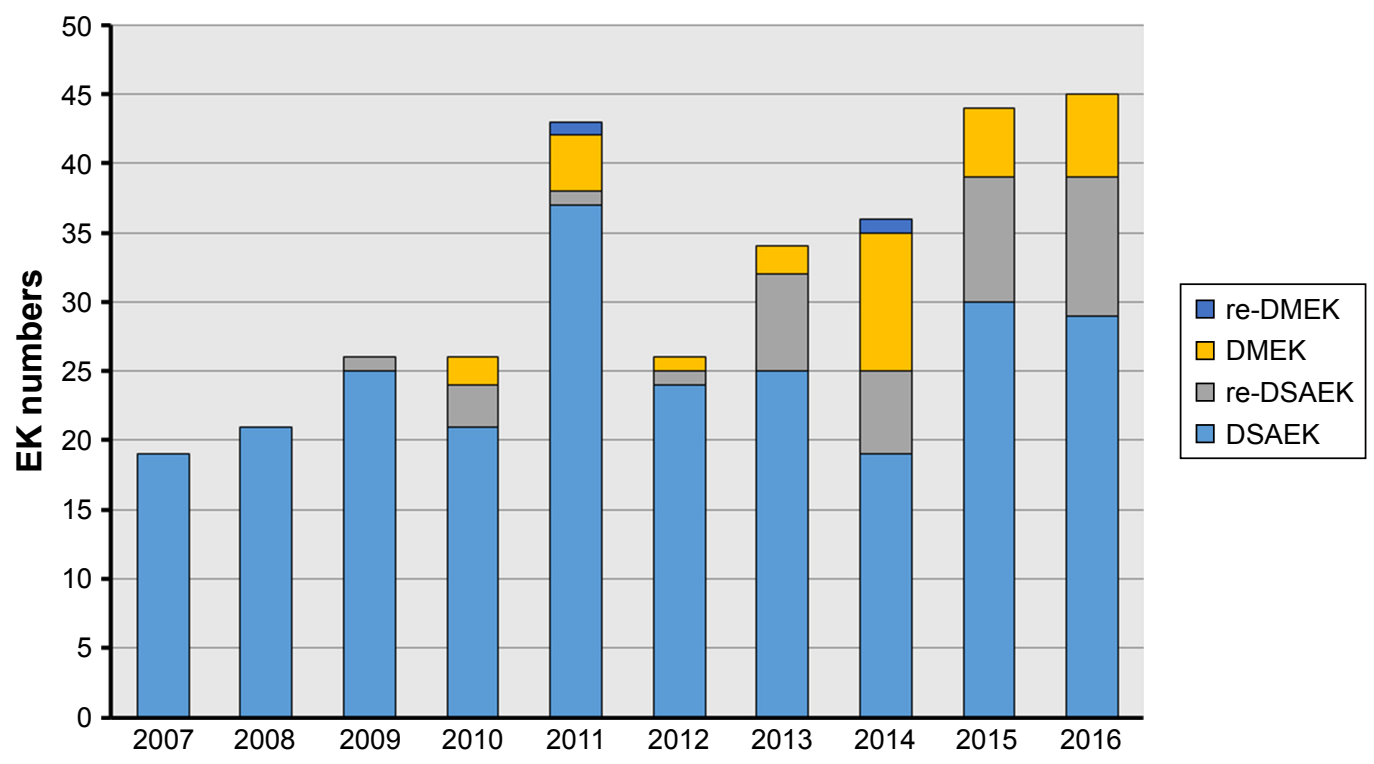

Figure I Surgical trends in EK over a 10-year period.

Notes: The total number of annual EK procedures gradually increased from 19 cases in 2007 to 45 cases in 20I6, with an exceptional spike in 201 I. DSAEK has consistently been the predominant type of EK.

Abbreviations: DMEK, Descemet's membrane endothelial keratoplasty; DSAEK, Descemet's stripping automated endothelial keratoplasty; EK, endothelial keratoplasty.

re-DSAEKs $(P<0.001)$ and DMEKs $(P<0.05)$ among all EK procedures increased during the same period (Figure 3, Table S1).

BK after ALI was the leading cause in 2007 (12 cases, $63.2 \%$ ), followed by FED (3 cases, 15.8\%) and failed PK ( 2 cases, 10.5\%). Ten years later in 2016, BK after TLE was the leading cause (12 cases, $26.7 \%$ ), followed by failed
DSAEK (10 cases, 22.2\%), failed PK (5 cases, 11.1\%), and PBK (5 cases, 11.1\%). The decrease in underlying ALI and FED between 2007 and 2016 was statistically significant $(P<0.001)$. In contrast, the increase in BK after TLE and failed DSAEK during the same period was also statistically significant $(P<0.001$; Figure 4 , Table S2). In terms of other minor indications, such as XFS, forceps delivery, failed

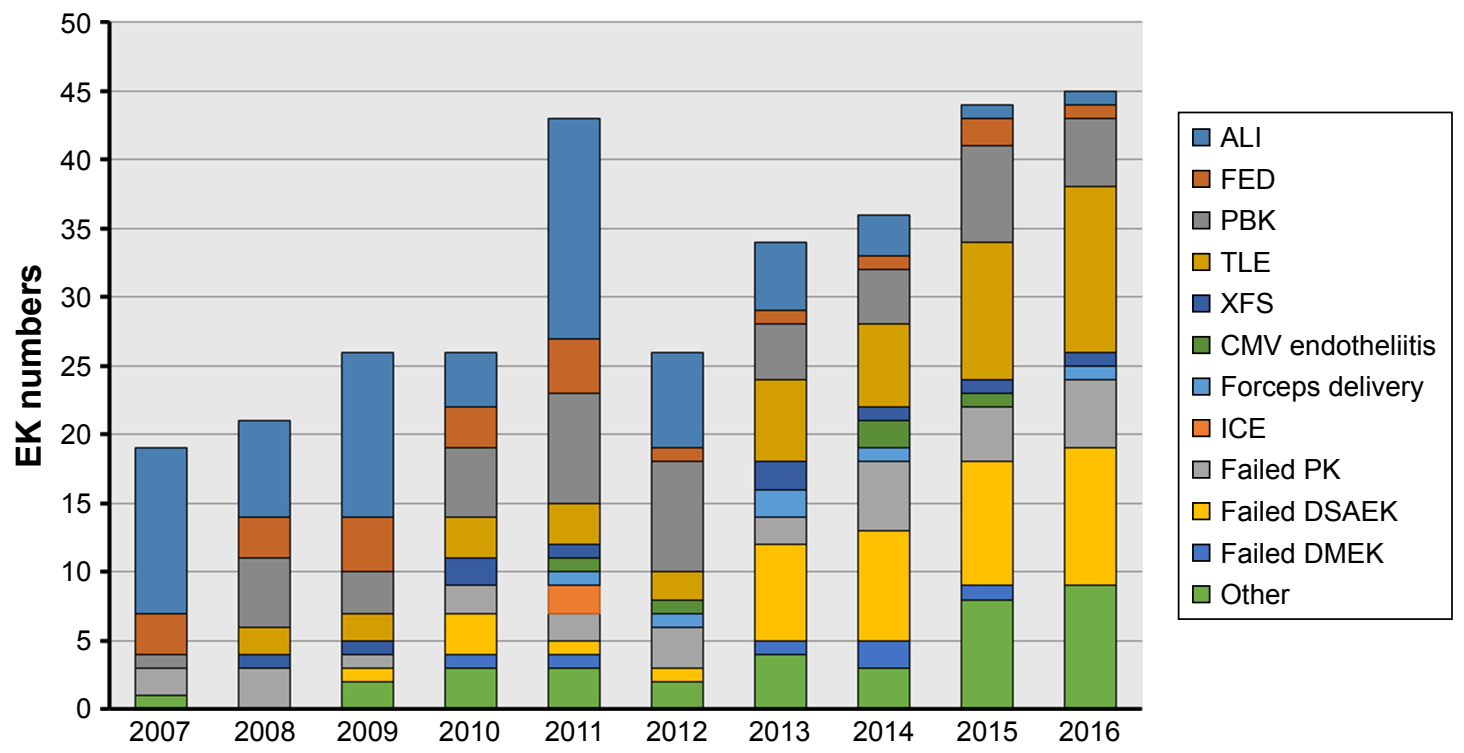

Figure 2 Annual trends in the number of endothelial keratoplasty procedures and their underlying diseases.

Note: Between 2007 and 2016, a decrease in BK after ALI and FED and an increase in BK after trabeculectomy and failed DSAEK was noted.

Abbreviations: ALI, argon laser iridotomy; BK, bullous keratopathy; CMV, cytomegalovirus; DMEK, Descemet's membrane endothelial keratoplasty; DSAEK, Descemet's stripping automated endothelial keratoplasty; FED, Fuchs' endothelial dystrophy; ICE, iridocorneal endothelial syndrome; PBK, pseudophakic BK; PK, penetrating keratoplasty; TLE, trabeculectomy; XFS, exfoliation syndrome. 


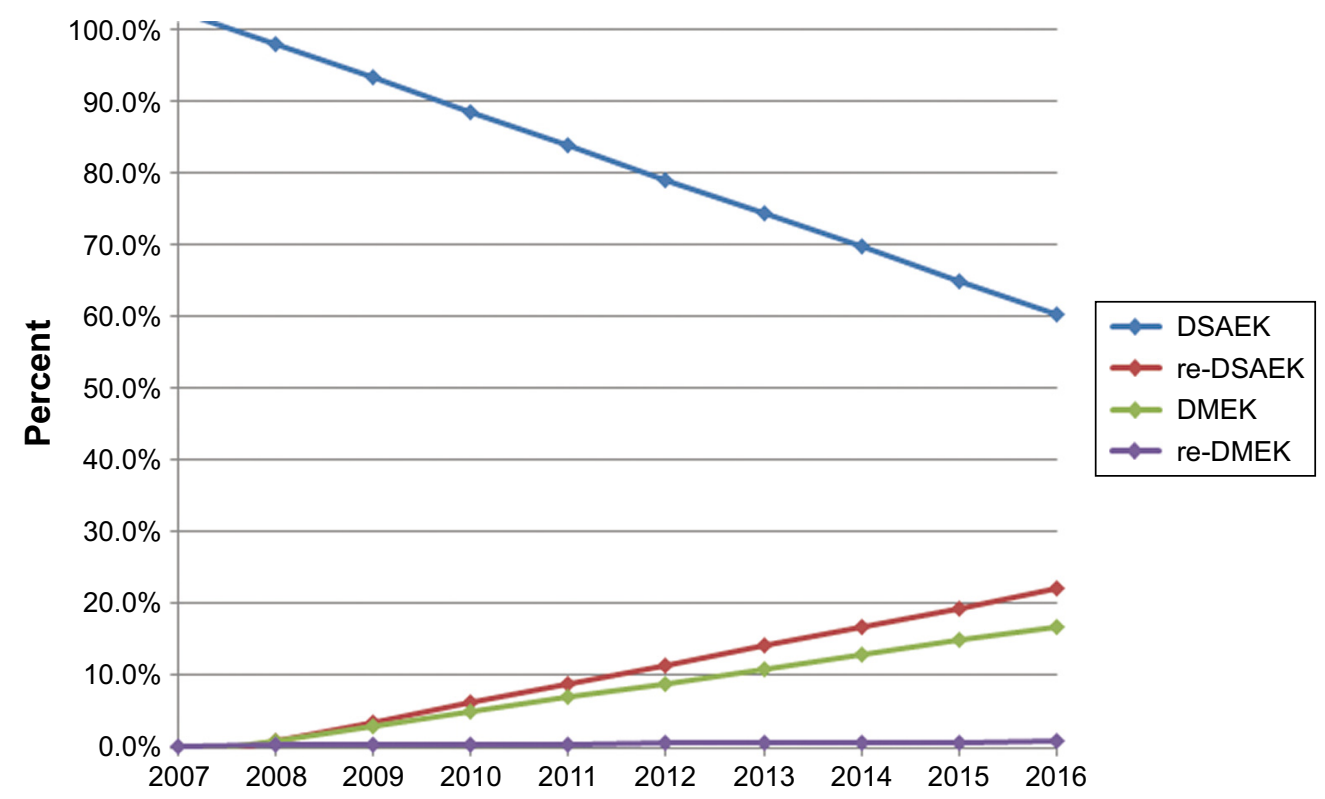

Figure 3 Changes in endothelial keratoplasty procedures over a 10-year period (2007-2016) using simple linear regression analysis.

Note: Significant annual trends were observed for three types of procedures, DSAEK, re-DSAEK, and DMEK. Slope for year indicates whether the proportion of each factor has changed over time, and the negative value corresponds to a declining trend over time.

Abbreviations: DMEK, Descemet's membrane endothelial keratoplasty; DSAEK, Descemet's stripping automated endothelial keratoplasty.

DMEK, CMV endotheliitis, and ICE, no significant changes were observed during the study period (Figure 4, Table S2).

\section{Discussion}

Currently, EK is the preferred surgical choice worldwide for the treatment of endothelial dysfunction. ${ }^{1-18}$ In the USA, EK has been the most common keratoplasty procedure for the last 5 years (DSAEK 77.2\%, DMEK 22.8\% in 2016). ${ }^{19}$
The leading indication for EK in the USA was FED (49.2\%), followed by PBK (17.2\%), and unknown/unreported cases (15.9\%). ${ }^{19}$ However, the reported causes of BK in Japan or Asia are different than those of western countries. ${ }^{23,24} \mathrm{We}$ specifically wanted to know the changes in EK procedures and underlying diseases. Results showed significant annual trends for 3 types of procedures, DSAEK, re-DSAEK, and DMEK. Although the total annual number of DSAEKs

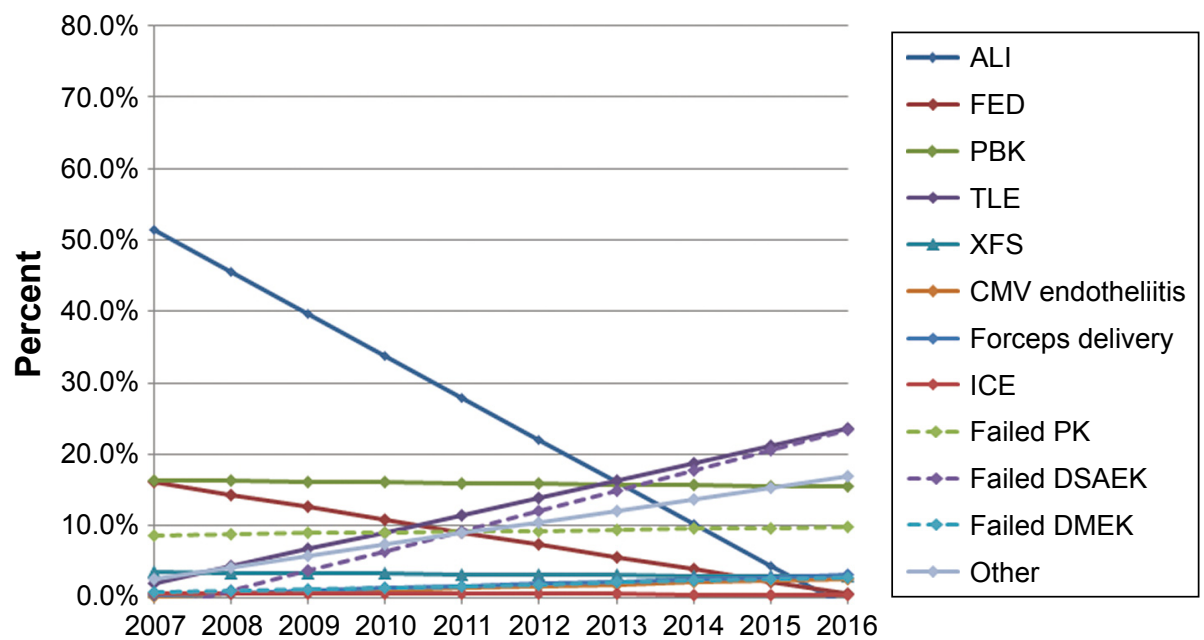

Figure 4 Changes in the underlying diseases of DSAEK/DMEK over a 10-year period (2007-2016) using simple linear regression analysis.

Notes: The decrease in underlying ALI and FED between 2007 and 2016 was statistically significant. In contrast, the increase in BK after TLE and failed DSAEK during the same period was also statistically significant. Slope for year indicates whether the proportion of each factor has changed over time, and the negative value corresponds to a declining trend over time.

Abbreviations: ALI, argon laser iridotomy; BK, bullous keratopathy; CMV, cytomegalovirus; DMEK, Descemet's membrane endothelial keratoplasty; DSAEK, Descemet's stripping automated endothelial keratoplasty; FED, Fuchs' endothelial dystrophy; ICE, iridocorneal endothelial syndrome; PK, penetrating keratoplasty; TLE, trabeculectomy; XFS, exfoliation syndrome. 
performed was stable between 2007 and 2016, the relative proportion of DSAEKs decreased significantly. In contrast, the proportion of re-DSAEKs and DMEKs increased among all EK procedures during the same period.

Additionally, we confirmed that the indications for EK in Japan are very different than in the USA or other western countries. Over the last 10 years, at our hospital, BK after ALI was the leading cause (21.3\%), followed by PBK (15.6\%), BK after TLE (14.4\%), failed DSAEK (12.5\%), and failed PK (9.1\%). It was somewhat surprising that FED as a cause of BK was as low as $7.2 \%$. Most significantly, we found that the indications for EK have changed over time; in 2007, BK after ALI was the leading cause (63.2\%), followed by FED (15.8\%) and failed PK (10.5\%). However, 10 years later in 2016, BK after TLE was the leading cause $(26.7 \%)$, followed by failed DSAEK (22.2\%), failed PK (11.1\%), and PBK (11.1\%). The decrease in ALI and FED and the increase in TLE and failed DSAEK during this period were statistically significant.

Previously, ALI was the favored treatment or preventive measure for acute angle-closure attack in Japan. However, some cases experienced late-onset endothelial dysfunction; ${ }^{23,24}$ although the precise mechanisms underlying BK after ALI remain unclear. For these reasons, laser iridotomy using yttrium aluminum garnet (YAG) lasers is currently used in Japan instead of ALI. Most recently, clearlens extraction has proven to be more cost-effective than laser peripheral iridotomy, and should be considered as an option for first-line treatment of angle-closure glaucoma. ${ }^{25}$ We conclude that these changes in treatment method from ALI to YAG and/or clear-lens extraction may be responsible for the recent reduction in the number of EK cases for BK after ALI.

As shown in this report, the annual number of DMEKs fluctuated from 2 to 10 cases over the 10-year span of this study; consequently, DSAEK has remained the predominant EK surgery at this hospital. The preference for DSAEK rather than DMEK is 2-fold. One reason is the difficulty of performing DMEK on the relatively smaller eyes with high vitreous pressure of Asian patients. Second, one of the primary indications for DMEK is FED, which is relatively rare in Japan; this is a well-known fact in Asia but may not be common knowledge to North American ophthalmologists. The difficulty of performing DMEK in Asian patients whose eyes have shallow anterior chambers with high vitreous pressure has been overcome by the development of new techniques and devices. We have previously reported the usefulness of an endoillumination probe for the visualization of DMEK grafts for Asian patients with dark brown irises. ${ }^{18}$ Moreover, the use of a standardized no-touch technique has helped to reduce complications during DMEK. ${ }^{26}$ The recent increase in BK after TLE and failed DSAEK may also have contributed to the increase in the number of DSAEK cases, since those eyes are usually difficult or contraindicated for DMEK.

The increased prevalence of TLE as an underlying disease can be explained by the fact that our hospital has begun to specialize in glaucoma over the years, and the number of patients with TLE has recently increased to as high as 300 cases per year. Furthermore, the increase in failed DSAEKs can simply be explained by the increase in recent DSAEK cases.

The limitation of the current report is that it is a single-center study and the results obtained herein may not necessarily reflect the overall trends in Japan as a whole. Nonetheless, this study has significance by showing the recent trends in a small area of Japan with a population of 1 million.

\section{Conclusion}

Changes in EK procedures (DSAEK/DMEK) and their underlying diseases over a 10-year period (2007-2016) at a tertiary referral hospital in Japan were noted. The proportion of re-DSAEK and DMEK procedures were increasing among all EK procedures. Most significantly, a decrease in ALI and FED cases and an increase in TLE and failed DSAEK were observed as underlying diseases for EK. Further analysis spanning a longer period of time and conducted as a multicenter study may be necessary to fully understand the changes in EK procedures and the causes of BK in Japan.

\section{Acknowledgment}

A Grant-in-Aid for Scientific Research (C) KAKENHI, Japan (No 16K11261).

\section{Disclosure}

The authors report no conflicts of interest in this work.

\section{References}

1. Melles GR, Eggink FA, Lander F, et al. A surgical technique for posterior lamellar keratoplasty. Cornea. 1998;17(6):618-626.

2. Terry MA, Ousley PJ. Small-incision deep lamellar endothelial keratoplasty (DLEK): six-month results in the first prospective clinical study. Cornea. 2005;24(1):59-65.

3. Terry MA, Ousley PJ. Deep lamellar endothelial keratoplasty visual acuity, astigmatism, and endothelial survival in a large prospective series. Ophthalmology. 2005;112(9):1541-1548.

4. Price MO, Price FW. Descemet's stripping with endothelial keratoplasty: comparative outcomes with microkeratome-dissected and manually dissected donor tissue. Ophthalmology. 2006;113(11):1936-1942.

5. Price FW, Price MO. Descemet's stripping with endothelial keratoplasty in 200 eyes: Early challenges and techniques to enhance donor adherence. J Cataract Refract Surg. 2006;32(3):411-418. 
6. Gorovoy MS. Descemet-stripping automated endothelial keratoplasty. Cornea. 2006;25(8):886-889.

7. Kobayashi A, Yokogawa H, Sugiyama K. Descemet stripping with automated endothelial keratoplasty for bullous keratopathies secondary to argon laser iridotomy - preliminary results and usefulness of doubleglide donor insertion technique. Cornea. 2008;27 Suppl 1:S62-S69.

8. Melles GR, Lander F, Rietveld FJ. Transplantation of Descemet's membrane carrying viable endothelium through a small scleral incision. Cornea. 2002;21(4):415-418.

9. Melles GR, Ong TS, Ververs B, van der Wees J. Descemet membrane endothelial keratoplasty (DMEK). Cornea. 2006;25(8):987-990.

10. Melles GR. Posterior lamellar keratoplasty: DLEK to DSEK to DMEK. Cornea. 2006;25(8):879-881.

11. Melles GR, Ong TS, Ververs B, van der Wees J. Preliminary clinical results of Descemet membrane endothelial keratoplasty. Am J Ophthalmol. 2008;145(2):222-227.

12. Ham L, Dapena I, van Luijk C, van der Wees J, Melles GR. Descemet membrane endothelial keratoplasty (DMEK) for Fuchs endothelial dystrophy: review of the first 50 consecutive cases. Eye (Lond). 2009; 23(10):1990-1998.

13. Ham L, Balachandran C, Verschoor CA, van der Wees J, Melles GR. Visual rehabilitation rate after isolated descemet membrane transplantation: Descemet membrane endothelial keratoplasty. Arch Ophthalmol. 2009;127(3):252-255.

14. Dapena I, Moutsouris K, Droutsas K, et al. Standardized "no-touch" technique for descemet membrane endothelial keratoplasty. Arch Ophthalmol. 2011;129(1):88-94.

15. Price MO, Giebel AW, Fairchild KM, Price FW. Descemet's membrane endothelial keratoplasty: prospective multicenter study of visual and refractive outcomes and endothelial survival. Ophthalmology. 2009; 116(12):2361-2368.

16. Anshu A, Price MO, Price FW Jr. Risk of corneal transplant rejection significantly reduced with Descemet's membrane endothelial keratoplasty. Ophthalmology. 2012;119(3):536-540.
17. Kobayashi A, Yokogawa H, Yamazaki N, Masaki T, Sugiyama K. In vivo laser confocal microscopy after Descemet's membrane endothelial keratoplasty. Ophthalmology. 2013;120(5):923-930.

18. Kobayashi A, Yokogawa H, Yamazaki N, Masaki T, Sugiyama K. The use of endoillumination probe-assisted Descemet membrane endothelial keratoplasty for bullous keratopathy secondary to argon laser iridotomy. Clin Ophthalmol. 2015;9:91-93.

19. Eye Bank Association of America. Eye Banking Statistical Report; 2016. Available from: www.restoresight.org

20. Terry MA, Shamie N, Chen ES, et al. Precut tissue for Descemet's stripping automated endothelial keratoplasty: vision, astigmatism, and endothelial survival. Ophthalmology. 2009;116(2):248-256.

21. Kobayashi A, Murata N, Yokogawa H, et al. Evaluation of internationally shipped prestripped donor tissue for descemet membrane endothelial keratoplasty by vital dye staining. Cornea. 2015;34(2):225-227.

22. Dobbins KR, Price FW Jr, Whitson WE. Trends in the indications for penetrating keratoplasty in the midwestern United States. Cornea. 2000;19(6):813-816.

23. Shimazaki J, Amano S, Uno T, et al. The Japan Bullous Keratopathy Study Group. National survey on bullous keratopathy in Japan. Cornea. 2007;26(3):274-278.

24. Ang LP, Higashihara H, Sotozono C, et al. Argon laser iridotomyinduced bullous keratopathy a growing problem in Japan. $\mathrm{Br} J$ Ophthalmol. 2007;91(12):1613-1615.

25. Azuara-Blanco A, Burr J, Ramsay C, et al. Effectiveness of early lens extraction for the treatment of primary angle-closure glaucoma (EAGLE): a randomised controlled trial. Lancet. 2016;388(10052): 1389-1397.

26. Monnereau C, Quilendrino R, Dapena I, et al. Multicenter study of descemet membrane endothelial keratoplasty: first case series of 18 surgeons. JAMA Ophthalmol. 2014;132(10):1192-1198. 


\section{Supplementary materials}

Table SI Changes in endothelial keratoplasty procedures over a 10-year period (2007-20I6) using simple linear regression analysis

\begin{tabular}{|c|c|c|c|c|c|}
\hline \multirow[t]{2}{*}{ Surgical procedure } & \multicolumn{3}{|c|}{ Slope for year* } & \multicolumn{2}{|c|}{ Intercept on 2007} \\
\hline & Estimate & $95 \% \mathrm{Cl}$ & $\overline{P \text {-value }}$ & Estimate & $95 \% \mathrm{Cl}$ \\
\hline DSAEK & $-4.70 \%$ & (-6.85\% to $-2.55 \%)$ & 0.001 & $102.56 \%$ & (91.08\% to $114.04 \%)$ \\
\hline re-DSAEK & $2.64 \%$ & ( $1.45 \%$ to $3.83 \%)$ & $<0.001$ & $-1.74 \%$ & (-8.10\% to $4.62 \%)$ \\
\hline DMEK & $1.99 \%$ & (0.38\% to $3.59 \%)$ & 0.022 & $-1.01 \%$ & $(-9.60 \%$ to $7.58 \%)$ \\
\hline re-DMEK & $0.07 \%$ & $(-0.22 \%$ to $0.36 \%)$ & 0.587 & $0.19 \%$ & (-I.33\% to $1.72 \%)$ \\
\hline
\end{tabular}

Note: *Slope for year indicates whether the proportion of each factor has changed over time, and the negative value corresponds to a declining trend over time. Abbreviations: DSAEK, Descemet's stripping automated endothelial keratoplasty; DMEK, Descemet's membrane endothelial keratoplasty.

Table S2 Changes in the underlying diseases of DSAEK/DMEK over a 10-year period (2007-2016) using simple linear regression analysis

\begin{tabular}{|c|c|c|c|c|c|}
\hline \multirow[t]{2}{*}{ Causal disease } & \multicolumn{3}{|c|}{ Slope for year* } & \multicolumn{2}{|c|}{ Intercept on 2007} \\
\hline & Estimate & $95 \% \mathrm{Cl}$ & $\overline{P \text {-value }}$ & Estimate & $95 \% \mathrm{Cl}$ \\
\hline$\overline{\mathrm{ALI}}$ & $-5.86 \%$ & $(-8.40 \%$ to $-3.32 \%)$ & $<0.001$ & $51.35 \%$ & (37.78\% to $64.92 \%)$ \\
\hline FED & $-1.72 \%$ & $(-2.28 \%$ to $-1.17 \%)$ & $<0.001$ & $16.02 \%$ & (13.06\% to $18.99 \%)$ \\
\hline PBK & $-0.09 \%$ & $(-2.09 \%$ to $1.91 \%)$ & 0.919 & $16.32 \%$ & (5.64\% to $27.00 \%)$ \\
\hline TLE & $2.40 \%$ & ( $1.44 \%$ to $3.37 \%)$ & $<0.001$ & $1.90 \%$ & $(-3.24 \%$ to $7.05 \%)$ \\
\hline XFS & $-0.06 \%$ & $(-0.72 \%$ to $0.59 \%)$ & 0.828 & $3.47 \%$ & $(-0.03 \%$ to $6.96 \%)$ \\
\hline CMV endothelitis & $0.27 \%$ & $(-0.22 \%$ to $0.77 \%)$ & 0.238 & $0.17 \%$ & $(-2.48 \%$ to $2.81 \%)$ \\
\hline Forceps delivery & $0.32 \%$ & $(-0.17 \%$ to $0.81 \%)$ & 0.169 & $0.26 \%$ & (-2.36\% to $2.88 \%)$ \\
\hline ICE & $-0.03 \%$ & $(-0.42 \%$ to $0.37 \%)$ & 0.873 & $0.59 \%$ & $(-1.52 \%$ to $2.70 \%)$ \\
\hline Failed PK & $0.12 \%$ & $(-0.86 \%$ to $1.11 \%)$ & 0.777 & $8.69 \%$ & (3.44\% to $13.94 \%)$ \\
\hline Failed DSAEK & $2.81 \%$ & (1.54\% to $4.08 \%)$ & $<0.001$ & $-1.94 \%$ & (-8.73\% to $4.84 \%)$ \\
\hline Failed DMEK & $0.23 \%$ & $(-0.27 \%$ to $0.74 \%)$ & 0.316 & $0.64 \%$ & $(-2.06 \%$ to $3.34 \%)$ \\
\hline Others & $1.60 \%$ & ( $0.68 \%$ to $2.52 \%)$ & 0.004 & $2.53 \%$ & $(-2.37 \%$ to $7.44 \%)$ \\
\hline
\end{tabular}

Note: *Slope for year indicates whether the proportion of each factor has changed over time, and the negative value corresponds to a declining trend over time.

Abbreviations: ALI, argon laser iridotomy; CMV, cytomegalovirus; DSAEK, Descemet's stripping automated endothelial keratoplasty; DMEK, Descemet's membrane endothelial keratoplasty; FED, Fuchs' endothelial dystrophy; ICE, iridocorneal endothelial syndrome; PBK, pseudophakic bullous keratopathy; PK, penetrating keratoplasty; TLE, trabeculectomy; XFS, exfoliation syndrome.

Clinical Ophthalmology

\section{Publish your work in this journal}

Clinical Ophthalmology is an international, peer-reviewed journal covering all subspecialties within ophthalmology. Key topics include: Optometry; Visual science; Pharmacology and drug therapy in eye diseases; Basic Sciences; Primary and Secondary eye care; Patien Safety and Quality of Care Improvements. This journal is indexed on

Submit your manuscript here: http://www.dovepress.com/clinical-ophthalmology-journal

\section{Dovepress}

PubMed Central and CAS, and is the official journal of The Society of Clinical Ophthalmology (SCO). The manuscript management system is completely online and includes a very quick and fair peer-review system, which is all easy to use. Visit http://www.dovepress.com/ testimonials.php to read real quotes from published authors. 